\title{
Naumann, Isabelle
}

\section{Von der Meritentafel zur Verhaltensampel - Lob und Strafe in der \\ Grundschule}

Thole, Friederike [Hrsg.]; Wedde, Sarah [Hrsg.]; Kather, Alexander [Hrsg.]: Über die Notwendigkeit der Historischen Bildungsforschung. Wegbegleiter*innenschrift für Edith Glaser. Bad Heilbrunn : Verlag Julius Klinkhardt 2021, S. 150-160

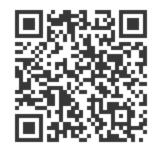

\section{Quellenangabe/ Citation:}

Naumann, Isabelle: Von der Meritentafel zur Verhaltensampel - Lob und Strafe in der Grundschule In: Thole, Friederike [Hrsg.]; Wedde, Sarah [Hrsg.]; Kather, Alexander [Hrsg.]: Über die Notwendigkeit der Historischen Bildungsforschung. Wegbegleiter*innenschrift für Edith Glaser. Bad Heilbrunn : Verlag Julius Klinkhardt 2021, S. 150-160 - URN: urn:nbn:de:0111-pedocs-233449 - DOI: 10.25656/01:23344

http://nbn-resolving.org/urn:nbn:de:0111-pedocs-233449

http://dx.doi.org/10.25656/01:23344

in Kooperation mit / in cooperation with:

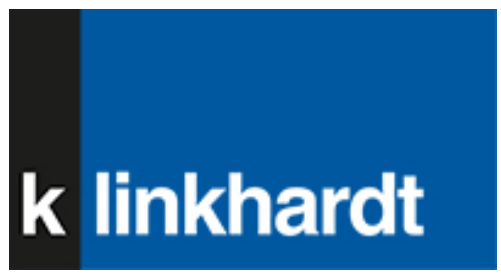

http://www.klinkhardt.de

\section{Nutzungsbedingungen}

Dieses Dokument steht unter folgender Creative Commons-Lizenz: http://creativecommons.org/licenses/by-nc-sa/4.0/deed.de - Sie dürfen das Werk bzw. den Inhalt unter folgenden Bedingungen vervielfältigen, verbreiten und öffentlich zugänglich machen sowie Abwandlungen und Bearbeitungen des Werkes bzw. Inhaltes anfertigen: Sie müssen den Namen des Autors/Rechteinhabers in der von ihm festgelegten Weise nennen. Dieses Werk bzw, der Inhalt darf nicht für kommerzielle Zwecke verwendet werden. Die neu entstandenen Werke bzw. Inhalte dürfen nur unter Verwendung von Lizenzbedingungen weitergegeben werden, die mit denen dieses Lizenzvertrages identisch oder vergleichbar sind.

Mit der Verwendung dieses Dokuments erkennen Sie die Nutzungsbedingungen an.

\section{Terms of use}

This document is published under following Creative Commons-License: http://creativecommons.org/licenses/by-nc-sa/4.0/deed.en - You may copy, distribute and transmit, adapt or exhibit the work in the public and alter, transform or change this work as long as you attribute the work in the manner specified by the author or licensor. You are not allowed to make commercial use of the work. If you alter, transform, or change this work in any way, you may distribute the resulting work only under this or a comparable license.

By using this particular document, you accept the above-stated conditions of use.

\section{Kontakt / Contact:}

\section{peDOCS}

DIPF | Leibniz-Institut für Bildungsforschung und Bildungsinformation Informationszentrum (IZ) Bildung

E-Mail: pedocs@dipf.de

Internet: www.pedocs.de

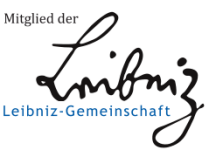




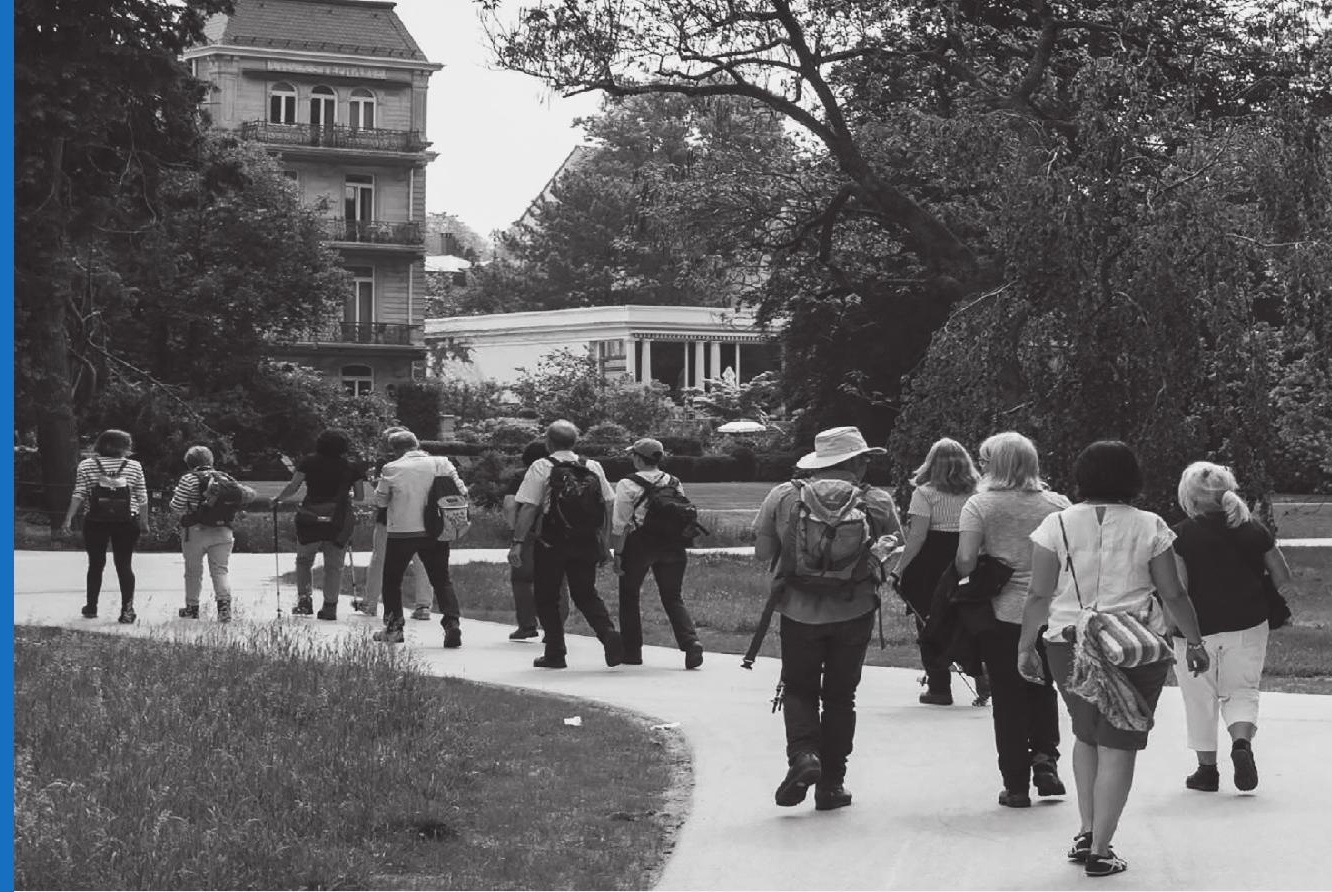

Friederike Thole

Sarah Wedde Alexander Kather

(Hrsg.)

Über die Notwendigkeit der Historischen Bildungsforschung

Wegbegleiter*innenschrift für Edith Glaser 


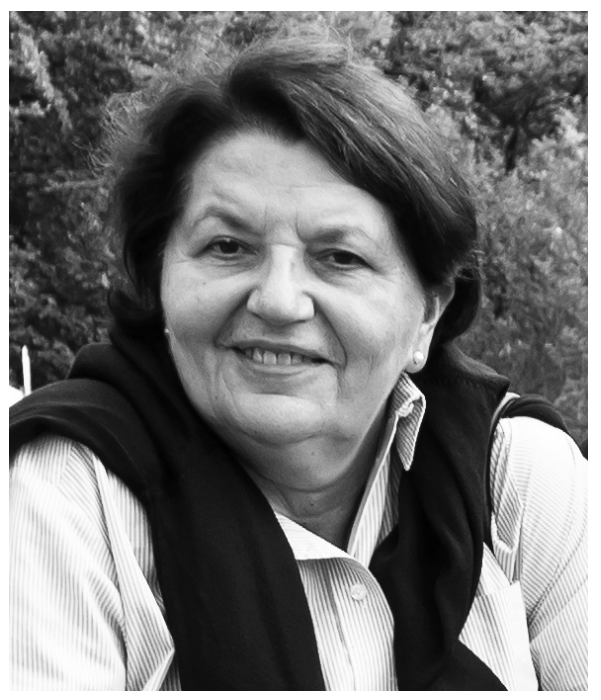

Edith Glaser ist seit 2006 Professorin für Historische Bildungsforschung an der Universität Kassel. Ihre Forschungsschwerpunkte sind die Disziplingeschichte der Erziehungswissenschaft, die historische Frauenund Geschlechterforschung sowie die geschichtliche Perspektive auf Bildungspolitik und Bildungsberatung. 


\section{Friederike Thole Sarah Wedde Alexander Kather (Hrsg.)}

\section{Über die Notwendigkeit der Historischen Bildungsforschung}

Wegbegleiter*innenschrift für Edith Glaser 
Dieser Titel wurde in das Programm des Verlages mittels eines Peer-Review-Verfahrens aufgenommen. Für weitere Informationen siehe www.klinkhardt.de.

Bibliografische Information der Deutschen Nationalbibliothek Die Deutsche Nationalbibliothek verzeichnet diese Publikation in der Deutschen Nationalbibliografie; detaillierte bibliografische Daten sind im Internet abrufbar über http://dnb.d-nb.de.

2021.n. () by Julius Klinkhardt.

Foto Umschlagseite 1: () Achim Heinrichs.

Druck und Bindung: AZ Druck und Datentechnik, Kempten.

Printed in Germany 2021.

Gedruckt auf chlorfrei gebleichtem alterungsbeständigem Papier.

(c) (i)(2) Die Publikation (mit Ausnahme aller Fotos, Grafiken und Abbildungen) ist veröffent-

licht unter der Creative Commons-Lizenz: CC BY-NC-SA 4.0 International

https://creativecommons.org/licenses/by-nc-sa/4.0/

ISBN 978-3-7815-5914-1 digital doi.org/10.35468/5914

ISBN 978-3-7815-2479-8 


\section{Inhaltsverzeichnis}

Friederike Thole, Sarah Wedde und Alexander Kather

Über die Notwendigkeit der Historischen Bildungsforschung -

Einführung in die Wegbegleiter*innenschrift für Edith Glaser

\section{Teil I}

Einblicke in ausgewählte Arbeitsbereiche der Historischen Bildungsforschung

Sarah Wedde und Friederike Thole

Historische Bildungsforschung schreibt Wissensgeschichte

\section{Ulrich Herrmann}

Die Bedeutung des Gemeinten im Gesagten.

Das Werkzeug des Historikers: Philologie und Hermeneutik -

am Beispiel von Wilhelm Flitners Veröffentlichungen in der NS-Zeit

Christian Bluhm und Markus Wochnik

Gender in der beruflichen Bildung - Entwicklung einer „Kategorie“

und einer zeitgemäßen Auseinandersetzung

\section{Pia Schmid}

Schule, Lehrerinnen und Lehrer in Autobiographien

politisch engagierter Frauen - 1850 bis 1910

Alexander Kather

„Wo die Quelle des Wissens ununterbrochen fließt“ -

Auslandsaufenthalte von Fremdsprachenlehrenden

aus (fach-)wissensgeschichtlicher Perspektive

Elke Kleinau

Children Born of War in der deutschen Nachkriegsgesellschaft -

Pädagogischer Diskurs und biografische Erzählungen 


\section{Teil II}

\section{Historische Bildungsforschung im inner- und interdisziplinären Kontext}

Ralf Mayer

,Zur Aufgabe des Erinnerns ‘ - im Spannungsfeld

von Orientierungsanspruch und Erinnerung

Werner Thole und Max-Ferdinand Zeterberg

Entdecken, Vergessen und Erinnern.

Über das ambivalente Rendezvous der Erziehungswissenschaft mit

der Sozialpädagogik bei Klaus Mollenhauer

Bernd Overwien

Wie politisch ist politische Bildung?

Kontroversen in der politischen Bildung seit den siebziger Jahren

Annedore Prengel

Anna und Maria mit dem Buch -

eine imaginierte pädagogische Beziehung

Friederike Heinzel und Julian Storck-Odabasi

100 Jahre Grundschule - Konzepte des Anfangsunterrichts

\section{Isabelle Naumann}

Von der Meritentafel zur Verhaltensampel -

Lob und Strafe in der Grundschule

Winfried Speitkamp

Vom Bauhaus lernen:

Das Staatliche Bauhaus von 1919 und die Universität der Zukunft 161

Verzeichnis der Autor*innen 


\title{
Von der Meritentafel zur Verhaltensampel - Lob und Strafe in der Grundschule
}

\author{
„Man darf, glaube ich, dreist behaupten, daß [sic!] in Ansehung keines Punkts bei der \\ Erziehung häufiger und gröblicher gefehlt werde, als in Ansehung der Belohnungen, \\ wodurch man Kinder und junge Leute zum Guten reizen, und der Strafen, \\ wodurch man sie von dem Bösen abzuschrecken sucht."
}

(Campe, 1788, zit. n. Blochmann, Geißler, Nohl \& Weniger, 1959, S. 27)

Den Ausgangspunkt dieses Beitrages markiert das Seminar „Erziehung und Strafe“, welches ich als Studentin des Grundschullehramts an der Universität Kassel im Wintersemester 2007/2008 besuchte. Edith Glaser führte in diesem ,in die historische und gegenwärtige Diskussion über Sinn und Angemessenheit von Strafe in der Erziehung sowie in die Veränderungen der Strafpraktiken in pädagogischen Verhältnissen" (Glaser, 2007) ein. Mit der im Seminar vorgestellten Meritentafel der Philanthropen setzte sie den Impuls, mich mit Erziehungsmitteln in der Grundschule intensiver auseinanderzusetzen. Die Reflexion eigener und beobachteter Belobigungs- und Bestrafungssysteme in der Schulpraxis mündete mehr als zehn Jahre später in gemeinsamen Lehrveranstaltungen mit Edith Glaser, in welchen das Thema Lob und Strafe in der pädagogischen Praxis aus bildungshistorischer und professionsethischer Perspektive gemeinsam mit den Studierenden aufgearbeitet und diskutiert wurde. Der vorliegende Beitrag greift diese Thematik auf und beleuchtet anhand studentischer Dokumentationen aktuell in der Grundschule als Erziehungsmittel angewandte Belobigungs- und Bestrafungssysteme sowie die Auseinandersetzung der Studierenden mit der vorgefundenen Praxis. Vorangestellt werden einige prominente Definitionsversuche des Erziehungsbegriffs sowie eine Rückschau auf historische Belobigungs- und Bestrafungssysteme in der Schule. Der Beitrag schließt mit einem Resümee zur Bedeutung der Historischen Bildungsforschung für die Reflexion gegenwärtiger pädagogischer Praktiken in der Grundschule auch im Setting schulpraktischer Studienanteile.

\section{Erziehung in Schule und Unterricht}

Der Strukturplan für das Bildungswesen weist Erziehung neben Lehren, Beurteilen, Beraten und Innovieren als Aufgabe von Lehrer*innen aus (vgl. Wissenschaftsrat, 1970, S. 12ff.) und in sämtlichen Schulgesetzen der deutschen Bundeslän- 
der finden sich Ausführungen zum Bildungs- und Erziehungsauftrag, wobei die Reihung der Begriffe variiert. Während die Zielsetzung, Schüler*innen im schulischen Kontext zu erziehen, damit als Selbstverständlichkeit erscheint, ist der Erziehungsbegriff vielfältig konnotiert (vgl. Dörpinghaus \& Uphoff, 2015, S. 19). Erziehung kann funktional als die „soziale Praxis des Umgangs der Menschen mit ihren Kindern“ (Wigger, 2012, S. 338) oder als „Reaktionen einer Gesellschaft auf die Entwicklungstatsache" (Bernfeld, 1928, S. 51) verstanden werden, womit eine "generationale Ordnung" (Heinzel, 2011, S. 44) des Erziehungsverhältnisses gesetzt zu sein scheint. Brezinka definiert Erziehung als intentionale „Handlungen [...], durch die Menschen versuchen, das Gefüge der psychischen Dispositionen anderer Menschen in irgendeiner Hinsicht dauerhaft zu verbessern oder seine als wertvoll beurteilten Bestandteile zu erhalten oder die Entstehung von Dispositionen, die als schlecht bewertet werden, zu verhüten" (Brezinka, 1990, S. 95), womit personale Aspekte und normative Orientierungen stärker betont werden. Der schulische Auftrag zur Erziehung ist in Abgrenzung zu dem der Bildung „vor allem auf das Verhalten und die moralischen Orientierungen der Heranwachsenden " (Horstkemper \& Tillmann, 2016, S. 20) ausgerichtet. Neben der Vermittlung von Kenntnissen, Fähigkeiten und Fertigkeiten sollen Schüler*innen in der Schule zur Anerkennung von gesellschaftlichen Wertordnungen befähigt und Lernbereitschaft, Leistungswille, Kooperations- und Konfliktfähigkeit sowie Toleranz, Gerechtigkeit und Solidarität gefördert werden (vgl. \$2 HSchG). Erziehende beziehungsweise Lehrer*innen sind dabei jedoch mit einem grundsätzlichen Paradoxon konfrontiert (vgl. Dörpinghaus \& Uphoff, 2015, S. 19): Während Erziehung einerseits auf Selbst- und Mitbestimmung ausgerichtet ist und das Kind als aktive*r Mitgestalter*in seiner Bildungsprozesse verstanden wird (vgl. HMSI \& HKM, 2019, S. 20), sind anderseits Grenzen, Regeln und bestehende Strukturen zu vermitteln, womit auch die Unterordnung des einzelnen Kindes an ebendiese einhergeht (vgl. Dörpinghaus \& Uphoff, 2015, S. 19). Zur Anpassung an schulische Verhaltensnormen können schulrechtlich legitimierte und klar definierte Ordnungsmaßnahmen als Reaktion auf unerwünschtes Schüler*innenverhalten zur Disziplinierung angewandt werden (vgl. \$82 HSchG). Pädagogische Maßnahmen im Unterricht, die möglichem Fehlverhalten vorbeugen sollen, sind hingegen bezüglich der konkreten Ausgestaltung in der Regel nicht näher bestimmt, womit Lehrer*innen ein weiter Handlungsspielraum zukommt (vgl. Stäsche, 2012, S. 340). Das Hessische Schulgesetz führt einige Interventionsmöglichkeiten auf, wie das Androhen von Ordnungsmaßnahmen, das Nachholen von Unterricht oder

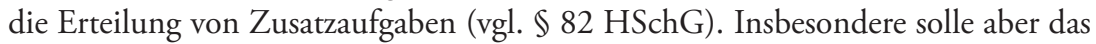
Gespräch mit dem*der Schüler*in gesucht werden, da pädagogische Maßnahmen „der Entwicklung des Lern- und Leistungswillens der Schülerin oder des Schülers und der Bereitschaft zu verantwortlichem sozialen Handeln nach den Grundsätzen der Toleranz, der Gerechtigkeit und der Solidarität dienen“ ( $\$ 82 \mathrm{HSchG}$ ) sollen. 


\section{Wettbewerb und Belobigung als schulische Erziehungsmittel}

Geißler unterscheidet direkte Erziehungsmittel, zu denen er Lob, Tadel, Ermahnung und Strafe zählt, sowie indirekte Erziehungsmittel wie Spiel, Wetteifer und Arbeit (vgl. Geißler, 1967, S. 20). Direkte und indirekte Erziehungsmittel sind jedoch nicht trennscharf zu denken, sondern mitunter ineinander verwoben. Die öffentlich praktizierte Belobigung, sichtbar gemacht durch eine Reihung der Sitzordnung - vorne die ,guten', hinten die ,schlechten ' Schüler ${ }^{1}$ - existierte bereits im römischen Schulwesen und diente dem Zweck, den Ehrgeiz unter den Schülern zu wecken (vgl. Herdegen, 2009, S. 30). Auch in den Jesuitenschulen, die sich ab dem ausgehenden 16. Jahrhundert entwickelten, waren „Wettkämpfe, Preisverleihungen und öffentliche Ehrungen [...], die die Jesuiten zu ihren wichtigsten Erziehungsmitteln zählten", (ebd., S. 37) zentrale Bestandteile. Durch die daraus resultierenden Rangfolgen und die Honorierung durch Ehrentitel und Preise wurde ein permanentes Konkurrenzstreben initiiert (vgl., ebd., S. 39ff.). Anreize zur Steigerung des Ehrgeizes wurden von den Philanthropen weiterentwickelt (vgl. Reh, Berdelmann \& Scholz, 2015, S. 41), wobei Fleiß und tugendhaftes Verhalten zu zentralen erzieherischen Absichten avancierten (vgl. Berdelmann, Reh \& Scholz, 2018, S. 145f.). Im Jahr 1777 führte Johann Bernhard Basedow die Meritentafel am Philanthropinum in Dessau als ausgeklügeltes Belobigungs- und Bestrafungssystem ein, welches öffentlich ausgehängt wurde (vgl. Sprengel, 1995, S. 7):

„Es werden Billetts gegeben für besonderen Fleiß, für vorzügliche Aufmerksamkeit und für gutes Verhalten, für Ordnung eigner Ausarbeitungen, für Wirkungen des besonderen Fleißes. 50 solche Billette gehören zu einem goldenen Punkt auf der Meritentafel, der nach der Gottesverehrung angeschlagen wird [...]. 50 Punkte geben einem Philanthropisten den Orden des Fleißes, der in einem Bande und einer silbernen Medaille besteht, welches er bei gewissen Feierlichkeiten tragen darf und welches macht, daß [sic!] er bei gewissen Umständen und Vergnügungen, daran nicht alle teilnehmen können, als Beordeter einen Vorzug hat.

Ein schwarzer Punkt wird erworben, wenn jemand zwölf schwarze Billette oder Striche bekommen hat, für zwölfmaliges Versehen. Doch ein Versehen mit sichtbar böser Absicht kann einige schwarze Bill. oder einen ganzen schwarzen Punkt zur Folge haben. Für beharrlichen Ungehorsam im Auftrage, der leicht ausgerichtet werden kann, wenn nicht der Wille fehlt, werden wohl 2 bis 3 schw. Billetts bestimmt und eine Nachricht darüber an die Eltern geschickt."

(Verzeichnis der den Philanthropisten zuerkannten und abgeschlagenen Billetts zur Erwerbung goldener Punkte auf den Meritentafeln, zit. n. Benner \& Kemper, 2000, S. 153).

1 Hier wird ausschließlich die männliche Form verwendet, da nur Knaben, Epheben und jungen Männern der Zugang zu institutionalisierten Bildung offenstand (vgl. Herdegen, 2009, S. 29). 
Anhand eines von Joachim Heinrich Campe verfassten Kriterienkatalogs zur Vergabe der Punkte und Billetts (vgl. Berdelmann, Reh \& Scholz, 2018, S. 146) oblag es am Ende jeder Unterrichtsstunde den Lehrern, über das Verhalten der ,Zöglinge' zu urteilen und dieses anhand von 20 Kurzzeichen in einem Meritenbuch zu dokumentieren (vgl. Sprengel, 1995, S. 7). Wenngleich die Meritentafel bei den Schülern ${ }^{2}$ sichtbare Wirkung erzielte, wurde diese im Kreise ihrer „Erfinder" aber auch kritisch hinterfragt. Campe führte Zweifel hinsichtlich der Auswirkungen auf das individuelle Wohl der Schüler an (vgl. Berdelmann, Reh \& Scholz, 2018, S. 148) und auch Karl Gottlieb Neuendorf, der das Philanthropinum zwischen 1784 und 1793 leitete, plädierte dafür, die Meritentafel durch andere Ermunterungen des Fleißes zu ersetzen (vgl. Sprengel, 1995, S. 7).

\section{Belobigungs- und Bestrafungssysteme in der Grundschule - ein exemplarischer Blick in die aktuelle Schulpraxis}

Zwecks eines Einblicks in die aktuelle pädagogische Praxis der Belobigung und Bestrafung in der Grundschule wird im Folgenden eine Dokumentenanalyse (vgl. Glaser, 2013) mittels einer sequenzanalytischen Rekonstruktion (vgl. Meseth, 2013) vorgenommen. Grundlage sind studentische Dokumentationen zu Belobigungs- und Bestrafungssystemen, die im Praxissemester des Lehramts an Grundschulen an der Universität Kassel im Wintersemester 2020/2021 entstanden. Die Studierenden wurden gebeten, die Belobigungs- und Bestrafungssysteme, die in ihren Praxissemesterklassen zum Einsatz kommen, zu dokumentieren. Insgesamt liegen 20 Texte vor, die unabhängig von einer Bearbeitung der Thematik im Seminar erstellt wurden. Die Durchsicht des Materials zeigt zunächst, dass in den Grundschulklassen eine Vielzahl an Belobigungs- und Bestrafungssystemen vorhanden ist. Beschrieben werden positive Verstärkungen durch verbales Lob von Lehrer*innen sowie Belohnungssysteme für einzelne Kinder oder Tischgruppen (z.B. Smileys, gesammelte Lobsteinchen oder Lobkarten wie „leisester Tisch") sowie individuelle oder kollektive Wochenziele, teils verbunden mit der gemeinschaftlichen Belobigung durch die Klasse (,jedes Kind, das einen Smiley erhält, bekommt einen Applaus von der Klasse"). Im Gegensatz dazu stehen aber auch vielfältige Maßnahmen, die zur Disziplinierung bei unerwünschtem Schüler*innenverhalten angewandt werden: Die Notation von Namen an der Tafel, die mit Strichen für Fehlverhalten versehen werden („Hat ein Kind drei Striche, werden die Eltern angerufen. "), die Vergabe gelber und roter Karten durch die Lehrer*innen, in deren Folge das Kind das eigene Fehlverhalten auf einem durch

2 Abgesehen von Basedows Tochter Emelie wurden am Dessauer Philanthropin ausschließlich Jungen unterrichtet (vgl. Rossini, 2015, S. 102). 
die Lehrkraft vorgefertigtes Regelblatt ankreuzen muss, die Separierung im „stillen Büro" in Form eines Tisches in der vorderen Ecke des Klassenraumes oder der Verweis vor die Klassentür. In mehr als einem Drittel der Dokumentationen taucht die sogenannte Verhaltensampel auf. Aus diesen Beschreibungen wurden zwei zusammengehörige Passagen ausgewählt, die im Folgenden vorgestellt und interpretiert werden, um die Studierendenperspektive auf die in der Praxisphase erlebten Belobigungs- und Bestrafungspraktiken zu rekonstruieren.

„Zum einen gibt es die Verhaltensampel. Diese besteht wie gewohnt aus dem grünen (lächelnder Smiley), dem gelben (neutraler Smiley) und dem roten Kreis (trauriger Smiley). Außerdem gibt es dazu jedoch noch einen silbernen Kreis. Die Namen der Kinder sind um diese Kreise herum mit Magneten angehangen, sodass das Bild einer Sonne entsteht. Am Anfang des Monats starten alle Kinder auf dem silbernen Kreis. Bei Unterrichtsstörungen oder Arbeitsverweigerungen (etc.) werden die betroffenen Kinder jeweils einzeln einen Kreis weiter gesetzt. Nach dem silbernen folgt der grüne, daraufhin der gelbe und schließlich der rote Kreis. Schafft ein Kind es den kompletten Monat auf Silber zu bleiben, bekommt dieses ein Lobkärtchen und darf sich etwas aus einer Überraschungskiste aussuchen. Wandert ein Kind auf Rot, bekommt dieses eine rote Karte. Diese enthält eine Benachrichtigung über das Verhalten des Schülers/ der Schülerin an die Eltern. Am nächsten Morgen, bevor der Unterricht beginnt, wandern alle Kinder, die den silbernen Kreis verlassen haben, zurück auf grün. Haben sie den silbernen Kreis einmal verlassen, haben sie erst im nächsten Monat wieder die Möglichkeit, dorthin zurück zu kommen. Die restlichen Felder starten jeden Tag aufs Neue. So erhält jedes Kind jeden Tag die Chance, es besser zu machen."

Durch die Eröffnung „zum einen“ wird zunächst darauf verwiesen, dass in dieser Klasse mehrere Belobigungs- und/oder Bestrafungssysteme eingesetzt werden. Die Existenz der Verhaltensampel wird mit dem Artikel „die“ eingeführt, womit eine allgemeine Kenntnis über selbige vorausgesetzt zu werden scheint. Dies bestätigt sich in der zweiten Sequenz: "wie gewohnt" besteht die Verhaltensampel aus Smileys in den Farben grün, gelb und rot. Hier wird eine Analogie zum Verkehrswechselzeichen der Ampel aufgemacht, deren farbliche Signale - grün steht für „freigegeben“, gelb für „warten“, rot für „halt“ - zur Verhaltensregulierung auf den schulischen Kontext übertragen werden und zusätzlich durch eine Rückmeldung auf der emotionalen Ebene (lächelnder, neutraler und trauriger Smiley) verstärkt werden. Ergänzt wird das dreifarbige Ampelsystem durch einen silbernen Kreis, dessen Position und Funktion zunächst nicht expliziert wird. Dieser ist auch nicht mit einer emotionalen Illustration versehen, sodass dessen Bedeutung offenbar für die Schüler*innen nicht visuell unterstrichen werden muss. Obwohl lediglich die Farben grün, gelb und rot im Straßenverkehr verbindliche Implikationen beinhalten, ist auch für den*die Verfasser*in der silberne Kreis zunächst nicht erklärungsbedürftig. Nachfolgend wird erkennbar, dass die Verhaltensampel nicht als System zur kollektiven Verhaltensregulation genutzt wird, sondern je- 
de*n Schüler*in einzeln adressiert. Inhärent ist dem System die Möglichkeit einer veränderten Positionierung, denn "[d]ie Namen der Kinder sind um diese Kreise herum mit Magneten angehangen", womit ohne großen Aufwand Veränderungen der Zuordnung von Kindern zu den Kreisen vorgenommen werden können. Der Turnus, in welchem sich die Schüler*innen auf der Verhaltensampel bewähren müssen, wird in der nachfolgenden Sequenz ersichtlich, wobei durch die Formulierung ,starten alle Kinder" bereits nahegelegt wird, dass ein wie auch immer geartetes Ziel zu erreichen ist, denn wer irgendwo startet, will oder muss auch meistens irgendwo ankommen. Den Ausgangspunkt markiert, wie wir nun wissen, der silberne Kreis. Eine Analogie zur Auszeichnung mit einer Medaille im Sport liegt hier sowohl in der Form als auch in der Farbe („silberne Kreis") nahe. Nachfolgend wird die Reihung der Kreise, es geht gestaffelt vom silbernen über den grünen und gelben Kreis ,schließlich“ und damit endgültig zum roten Kreis, erläutert. Die Verhaltensampel scheint mit einer Herausforderung verbunden zu sein, denn es gilt, dass etwas über einen längeren Zeitraum „[ge]schafft“ werden muss. Dass es sich um einen „kompletten Monat" handelt, ist insofern beachtenswert, dass es sich hierbei nicht um eine typische schulische Zeiteinheit handelt. Vielmehr scheint die Dauer eines Monats ergänzend zu kurzen (Stundenplan/ Wochenplan) und längeren schulischen Zeiteinteilungen (Halbjahr/Schuljahr) einen zusätzlichen und damit besonderen Fixpunkt zu markieren. Im Zuge dieser Sequenz wird nun auch das Ziel ersichtlich: Die Schüler*innen erhalten eine Belobigung, wenn sie die Herausforderung gemeistert haben, den Unterricht nicht zu stören und auf dem silbernen Kreis zu verweilen. Auf die Belobigung, in der sprachlichen Variation als Diminutiv („Lobkärtchen“) im Gegensatz zur gelben und roten Karte verniedlichend dokumentiert, folgt eine materielle Belohnung, deren Wert durch die Zugänglichkeit zur „Überraschungskiste“ gesteigert wird. Die Belohnung ist zudem mit einem nicht-materiellen Gewinn verbunden, nämlich dem zugestandenen Privileg der Entscheidungsfreiheit („darf sich etwas aus der Überraschungskiste aussuchen"). Der zweimalige Verweis auf das Wandern der Kinder auf der Verhaltensampel legt beinahe eine scheinbar vergnügliche Eigenaktivität nahe. Unklar bleibt, wer über die Positionen an der Verhaltensampel entscheidet. Die Einführung eines abermals aus dem Sport entliehenen Symbols, der roten Karte, die durch eine*n Unparteiische*n, den*die Schiedsrichter*in vergeben wird, verweist jedoch auf eine entscheidende, sich damit als objektiv inszenierende Instanz, vermutlich die Lehrkraft. Verbunden wird die rote Karte, die im Sport einen Ausschluss aus dem Spielgeschehen zur Folge hat, hier mit der Hinzuziehung einer weiteren Erziehungsinstanz, den Eltern. Die zusätzlichen Konsequenzen, die möglicherweise auf das „Fehlverhalten“ der Schüler*innen folgen, werden somit von der Schule in den privaten Raum delegiert. Indem alle Kinder täglich zu Beginn des Unterrichts wieder auf dem grünen Kreis starten, wird der Kreislauf der Verhaltensbewährung und Disziplinierung permanent er- 
neut in Gang gesetzt, hier positiv konnotiert durch die behauptete Chance, dass es ,jedes Kind jeden Tag [...] besser [...] machen“" kann. Dabei bleibt jedoch allen, die sich auf dem grünen Kreis befinden, die Chance bis zu Beginn des nächsten Monats verwehrt, wieder auf das höchste Belobigungsniveau, den silbernen Kreis, zurückzukehren. Die Position auf dem silbernen Kreis wird damit nochmals als besonders wertvoll herausgestellt und betont, dass diese durch einmaliges Fehlverhalten dauerhaft verwirkt wird. Ergänzend zum Text war der studentischen Dokumentation ein Foto der Verhaltensampel beigefügt, welches hier aufgrund der Angabe der Namen und einer möglichen Zuordnung zu einer bestimmten Klasse aus datenschutzrechtlichen Gründen nicht gezeigt wird. Auf diesem Bild sind alle Kinder am grünen Kreis positioniert, so dass ersichtlich wird, das jedes Kind in dem entsprechenden Monat mit Hilfe der Verhaltensampel diszipliniert wurde. Nachfolgend wird nun die studentische Auseinandersetzung mit der vorgefundenen Praxis der Verhaltensampel in den Blick genommen. Grundlage ist die Kommentierung des Belobigungs- und Bestrafungssysteme hinsichtlich des pädagogischen Nutzens sowie der damit verbundenen Vor- und Nachteile.

„Ich persönlich finde das Ampelsystem sehr sinnvoll. Die Kinder werden während des Unterrichts immer wieder daran erinnert, sich entsprechend der Regeln, welche sie alle kennen, zu verhalten. Dazu muss die Lehrkraft nicht viel zu beitragen, da dies automatisch geschieht, da die Kinder immer einen Blick auf die Ampel neben der Tafel haben. Vereinzelte Kinder testen trotzdem immer wieder ihre Grenzen aus und gehen über Ermahnungen hinaus. Dabei ist jedoch zu beobachten, dass dies aufhört, sobald sie am gelben Kreis angekommen sind. Vermutlich, weil der rote Kreis mit einer Konsequenz verbunden ist. Daher ist dieses System aus meiner Perspektive sehr nützlich."

Es zeigt sich, dass der*die Verfasser*in die Verhaltensampel uneingeschränkt befürwortet, was durch das eingangs verwendete Adverb ,sehr“ in Verbindung mit dem Adjektiv „sinnvoll“ deutlich wird. Der Nutzen der Verhaltensampel wird rein zweckorientiert begründet, indem die Erinnerung an die Regeln („Die Kinder werden während des Unterrichts immer wieder daran erinnert") sowie die Entlastung der Lehrkraft („Dazu muss die Lehrkraft nicht viel zu beitragen, da dies automatisch geschieht") als Argumentationslinie für die Zustimmung herangezogen werden. Es kommt durch die Omnipräsenz der Verhaltensampel neben der Tafel, auf welche die Schüler*innen quasi permanent ausgerichtet zu sein scheinen, automatisch, also ohne Einwirkung einer*s Akteurs*in, zu einer Verhaltensregulation - so die Darstellung der*des Studierenden. Vorausgesetzt wird dabei, dass die Regeln, an denen die Kinder gemessen werden, Allgemeingültigkeit besitzen und allen bekannt sind. Durch die Formulierung "[v]ereinzelte Kinder testen trotzdem immer wieder ihre Grenzen aus" wird jeglicher Regelübertritt als aktiver und bewusster Akt der Schüler*innen interpretiert. Zugleich wird die Lehrkraft indirekt entlastet, weil eben kein subjektiver Akt der Wertung mit dem Strafen verbunden 
wird, sondern die Durchsetzung von Grenzen und Regeln von allgemeinem Bekanntheitsgrad. Man könnte auch sagen, die hier tätigen Lehrer*innen tun nur ihre Pflicht, eine falsche Einschätzung beziehungsweise eine fälschlicherweise vollzogene Einordnung auf der Verhaltensampel bleibt als Möglichkeit aus. Nochmals wird argumentiert, dass die Aussicht auf eine Konsequenz, nämlich der Einbezug der Eltern, wirkungsvoll ist und das System damit als Ganzes als „sehr nützlich“ legitimiert.

\section{Fazit}

Die studentischen Dokumentationen geben einen exemplarischen Einblick in pädagogische Maßnahmen in der Grundschule in Form von Belobigungs- und Bestrafungssystemen. Indem durch den Blickwinkel Studierender „etwas zur Sprache gebracht wird, was vorher nicht Sprache war" (Hirschauer, 2001, S. 430), wird gezeigt, dass der traditionell wertende Verhaltensvergleich zwischen Schüler*innen noch immer fest verankerter Bestandteil von Schule ist. Die historische Meritentafel und die gegenwärtige Verhaltensampel stellen Disziplinierungspraktiken dar, mittels derer in erster Line durch das In-Verhältnis-Setzen und die soziale Bezugnahme versucht wird, auf das Verhalten der* $^{*}$ des Einzelnen einzuwirken (vgl. Wolter, 2018, S. 99). Anpassung und Verstoß der Schüler*innen gegen die geltenden Regeln werden noch immer öffentlich sichtbar gemacht und diese damit individuell zur Disposition gestellt. Schulische Sanktionsmittel wie Lob, Tadel und Strafe haben damit

„erklärtermaßen und institutionell gesichert die Bedeutung, etwas über die Person dessen auszusagen, auf den sie gemünzt sind. Sie werden zudem öffentlich erteilt im Angesicht der Schulklasse, und es ist dafür gesorgt, daß [sic!] den Eltern [...] Verweise nicht verborgen bleiben. [...] Das Gefühl persönlicher Unzulänglichkeiten und die individuelle Selbstachtung werden so zum Ansatzpunkt der Verhaltenssanktionierung (vgl. Lenhardt, 1984, S. 199).

Im vorliegenden Beispiel war der Blick der*des Studierenden nicht auf das Kind und dessen emotionales Befinden gerichtet, sondern ausschließlich auf die der Verhaltensampel attestierten Vorteile hinsichtlich der Aufrechterhaltung des Unterrichtsgeschehen. Schulische Regeln und damit verbundene Disziplinierungsmaßnahmen dienen im gezeigten Fallbeispiel in hohem Maße der Normierung der Schüler*innen. Wettbewerb und Vergleich werden instrumentalisiert, damit die Schüler*innen ,funktionieren' und auf institutionalisierte Signale möglichst schnell reagieren. Dies muss anhand einer weiteren studentischen Dokumentation untermauert werden, in welcher konstatiert wird, dass der größte Vorteil der 
Vergabe einer roten Karte darin liege, dass dies „stumm geschehen kann und nicht thematisiert wird“, „das Unterrichtsgeschehen nicht großartig durch Diskussionen und Rechtfertigungen unterbrochen wird“, weswegen die „Umsetzung im Unterricht [...] als angehende Lehrerin sehr attraktiv" sei. Wenngleich das Ziel, Schüler*innen Regeln für die Gemeinschaft in und außerhalb der Schule zu vermitteln, hier nicht negiert werden soll, ist doch darauf aufmerksam zu machen, dass aus der studentischen Perspektive heraus in beiden Beispielen nicht diskutiert wird, inwiefern das angewandte Belobigungs- und Bestrafungssystem andere Bildungs- und Erziehungsziele, wie etwa die freie Entfaltung der Persönlichkeit sowie Toleranz, Gerechtigkeit und Solidarität in der Gemeinschaft (vgl. $\$ 2$ HSchG) konterkariert. Kritische Stimmen zu Praxisphasen verweisen auf „unerwünschte[n] Lerneffekte von Praktika, die aus einer unreflektierten Adaption einer zumindest teilweise problematischen Schul- und Unterrichtspraxis resultieren“" (Bach, 2013, S. 148) beziehungsweise „einseitige Prozesse des Anpassungsverhaltens der Studierenden an die Lehrpersonen“ (Hascher, 2012, S. 94). Am vorliegenden Beispiel der Beschreibung und Kommentierung der Verhaltensampel bestätigt sich eine unreflektierte Zustimmung zum Handeln der Lehrkraft in der Praktikumsklasse. Auch in der Gesamtschau aller Dokumentationen sind kritisch-reflektierende Überlegungen nur marginal erkennbar. Selbst stark bloßstellende und ausschließende Disziplinierungsmaßnahmen (vgl. Reckahner Reflexionen, 2017), wie der Zwang zum Sitzen an einem exponierten Tisch („stilles Büro“) oder der Verweis aus dem Klassenraum, werden nicht mit Blick auf das Befinden der Schüler*innen reflektiert. Die Gegenüberstellung der Meritentafel und der Verhaltensampel zeigt deutliche Parallelen und lässt eine erstaunliche Stabilität erzieherischer Praktiken in der Schule erkennen: Die öffentliche Darstellung der Erfüllung oder des Verstoßes der institutionell gesetzten Verhaltensnormen, die Sammlung von Bewertungen über eine längere Dauer (50 Billetts ergeben goldenen Punkt versus Lobkärtchen bei komplettem Monat auf silbernem Kreis), die Information bei Verstoß an die Eltern (Nachricht bei zwei bis drei schwarzen Billetts versus Benachrichtigung über Verhalten, wenn ein Kind auf den roten Kreis kommt), die symbolische Auszeichnung mit besonders wertvollem Edelmetall (goldener Punkt und silberne Medaille versus silberner Kreis) und zuletzt ein abschließendes Vorzugsrecht ,Belobigter ' gegenüber den Mitschüler*innen (Orden des Fleißes - Privileg der Teilnahme an Feierlichkeit versus Lobkärtchen - Privileg des Zugriffs auf die Überraschungskiste).

In den studentischen Dokumentationen ist in doppelter Hinsicht Potential verborgen. Zum einen retrospektiv aus einer Forscher*innenperspektive, da diese der erziehungswissenschaftlichen Forschung den Blick auf schulische Erziehungspraktiken eröffnen (vgl. Glaser, 2013, S. 366), die bislang theoretisch und empirisch wenig bearbeitet sind (vgl. Wolter, 2018, S. 100). Zum anderen kann weiterführend die Auseinandersetzung mit historischen (Erziehungs-)Praktiken in der Schule im Rahmen praxisbegleitender Lehrveranstaltungen als bildungshistorisches 
Orientierungswissen (vgl. Glaser, 2009, S. 75) dafür genutzt werden, die erlebte Schulpraxis "forschungs- und nicht subjektiv-erfahrungsfundiert" zu analysieren und zu reflektieren, um „bewährte Routinen und Selbstverständlichkeiten zu bewerten und gegebenenfalls zu hinterfragen sowie das eigene Handeln wie die Handlungsroutinen anderer [...] in der Einzelschule vor Ort zu prüfen" (Rothland \& Bennewitz, 2018, S. 38), denn „[e]rst aus der Kenntnis der Grundschulgeschichte können aktuelle Problemstellungen, Diskussionen und Entwicklungen angemessen eingeschätzt werden" (Götz \& Sandfuchs, 2014, S. 43).

\section{Quellen}

Verzeichnis der den Philanthropisten zuerkannten und abgeschlagenen Billetts zur Erwerbung goldener Punkte auf den Meritentafeln - Abteilung I/1 der Reliquiae Philanthropini, 2. Band (abgedruckt bei Benner \& Kemper, 2000, S. 153).

\section{Literatur}

Bach, Andreas (2013). Kompetenzentwicklung im Schulpraktikum. Ausmaß und zeitliche Stabilität von Lerneffekten hochschulischer Praxisphasen. Münster: Waxmann.

Benner, Dietrich \& Kemper, Herwart (2000). Quellentexte zur Theorie und Geschichte der Reformpädagogik. Teil 1: Die pädagogische Bewegung von der Aufklärung bis zum Neuhumanismus. Weinheim: Deutscher Studien Verlag.

Berdelmann, Kathrin, Reh, Sabine \& Scholz, Joachim (2018). Wettbewerb und Ehrtrieb. Die Entstehung des Leistungs-Dispositivs im Schulwesen um 1800. In Sabine Reh \& Norbert Ricken (Hrsg.), Leistung als Paradigma. Zur Entstehung und Transformation eines pädagogischen Konzepts. Wiesbaden: Springer VS, S. 137-163.

Bernfeld, Siegfried (1928). Sisyphos oder die Grenzen der Erziehung (2. Aufl.). Leipzig/Wien/ Zürich: Internationaler Psychoanalytischer Verlag.

Blochmann, Elisabeth, Geißler, Georg, Nohl, Herrmann \& Weniger, Erich (1959). Die Strafe in der Erziehung. Kleine Pädagogische Texte. Band 24. Weinheim: Beltz.

Brezinka, Wolfgang (1990). Grundbegriffe der Erziehungswissenschaft. Analyse, Kritik, Vorschläge (5., verb. Aufl.). München/Basel: Ernst Reinhardt Verlag.

Dörpinghaus, Andreas \& Uphoff, Ina Katharina (2015). Grundbegriffe der Pädagogik (4. Aufl.). Darmstadt: WBG.

Geißler, Erich E. (1967). Erziehungsmittel. Bad Heilbrunn: Verlag Julius Klinkhardt.

Glaser, Edith (2007). Erziehung und Strafe. Vorlesungsverzeichnis Universität Kassel Wintersemester 2007/08. https://portal.uni-kassel.de/qisserver/rds?state=verpublish\&status=init\&vmfile=no\&publishid=25492\&moduleCall=webInfo\&publishConfFile=webInfo\&publishSubDir=veranstaltung

Glaser, Edith (2009). Aus Bildungsgeschichte(n) lernen. Zeitschrift für pädagogische Historiographie 15(2), S. 74-75.

Glaser, Edith (2013). Dokumentenanalyse und Quellenkritik. In Barbara Friebertshäuser, Antje Langer \& Annedore Prengel (Hrsg.), Handbuch Qualitative Forschungsmethoden in der Erziehungswissenschaft (4. Aufl.). Weinheim u.a.: Beltz Juventa, S. 365-375.

Götz, Margarete \& Sandfuchs, Uwe (2014). Geschichte der Grundschule. In Wolfgang Einsiedler, Margarete Götz, Andreas Hartinger, Friederike Heinzel, Joachim Kahlert \& Uwe Sandfuchs (Hrsg.), Handbuch Grundschulpädagogik und Grundschuldidaktik (4. Aufl.). Bad Heilbrunn: Verlag Julius Klinkhardt, S. 32-45. 


\section{Isabelle Naumann}

Hascher, Tina (2012). Forschung zur Bedeutung von Schul- und Unterrichtspraktika in der Lehrerinnen- und Lehrerbildung. Beiträge zur Lehrerinnen- und Lehrerbildung, 30(1), S. 87-98.

Heinzel, Friederike (2011). Kindgemäßheit oder Generationenvermittlung als grundschulpädagogisches Prinzip? In Friederike Heinzel (Hrsg.), Generationenvermittlung in der Grundschule. Ende der Kindgemäßßheit? Bad Heilbrunn: Verlag Julius Klinkhardt, S. 40-68.

Herdegen, Peter (2009). Schulische Prüfungen. Entstehung - Entwicklung - Funktion. Prüfungen am bayerischen Gymnasium vom 18. bis zum 20. Jahrhundert. Bad Heilbrunn: Verlag Julius Klinkhardt.

Hessisches Ministerium für Soziales und Integration (HMSI) \& Hessisches Kultusministerium (HKM) (2019). Bildung von Anfang an. Bildungs- und Erziehungsplan für Kinder von 0 bis 10 Jahren in Hessen (9. Aufl.). Wiesbaden.

Hessisches Schulgesetz (HSchG) in der Fassung vom 30. Juni 2017. https://kultusministerium.hessen. de/schulsystem/schulrecht

Hirschauer, Stefan (2001). Ethnographisches Schreiben und die Schweigsamkeit des Sozialen. Zu einer Methodologie der Beschreibung. Zeitschrift für Soziologie, 30(6), S. 429-451.

Horstkemper, Marianne \& Tillmann, Klaus-Jürgen (2016). Sozialisation und Erziehung in der Schule. Eine problemorientierte Einführung. Bad Heilbrunn: Verlag Julius Klinkhardt.

Lenhardt, Gero (1984). Schule und bürokratische Rationalität. Frankfurt a. M.: Suhrkamp Verlag.

Meseth, Wolfgang (2013). Die Sequenzanalyse als Methode einer erziehungswissenschaftlichen Empirie pädagogischer Ordnungen. In Barbara Friebertshäuser \& Sabine Seichter (Hrsg.), Qualitative Forschungsmethoden in der Erziehungswissenschaft. Eine praxisorientierte Einführung. Weinheim u.a.: Beltz Juventa, S. 63-80.

Reh, Sabine, Berdelmann, Kathrin \& Scholz, Joachim (2015). Der Ehrtrieb und unterrichtliche Honorierungspraktiken im Schulwesen um 1800. In Alfred Schäfer \& Christiane Thompson (Hrsg.), Leistung. Paderborn: Schöningh, S. 37-60.

Reckahner Reflexionen zur Ethik pädagogischer Beziehungen. Hrsg. von Deutschen Institut für Menschenrechte, Deutschen Jugendinstitut e. V., MenschenRechtsZentrum an der Universität Potsdam, Rochow-Museum und Akademie für bildungsgeschichtliche und zeitdiagnostische Forschung e. V. an der Universität Potsdam in 2017. Rochow-Edition: Reckahn.

Rossini, Sara (2015). „Wie kommts, daß es mit den Philanthropinen so gar nicht fort will?“ Die Kritik an den Erziehungsmethoden der Philanthropen in fiktionalen Texten der Aufklärung. Colmbus: Ohio State University.

Rothland, Martin \& Bennewitz, Hedda (2018). Praktiker zu Theoretikern!? Das Schulpraxiserfordernis oder warum Ewald Terhart kein Schulpädagoge sein dürfte. In Martin Rothland \& Manfred Lüders (Hrsg.), Lehrer-Bildungs-Forschung; Festschrift für Ewald Terhart. Münster/New York: Waxmann, S. $25-41$.

Sprengel, Günter (1995). 50 Punkte braucht der Philanthropist für den Fleißorden. Die Meritentafel des Dessauer Philanthropinum zeigt ein ausgeklügeltes Bewertungssystem. Deutsche Lehrerzeitung, 42(7), S. 7.

Stäsche, Uta (2012). Erziehungs- und Ordnungsmaßnahmen. In Klaus-Peter Horn, Heidemarie Kemnitz, Winfried Marotzki \& Uwe Sandfuchs (Hrsg), Klinkhardt Lexikon Erziehungswissenschaft. KLE Band 1. Aa, Karl von der-Gruppenprozesse. Bad Heilbrunn: Verlag Julius Klinkhardt, S. 340-341.

Wigger, Lothar (2012). Erziehung. In Klaus-Peter Horn, Heidemarie Kemnitz, Winfried Marotzki \& Uwe Sandfuchs (Hrsg.), Klinkhardt Lexikon Erziehungswissenschaft. KLE Band 1. Aa, Karl von derGruppenprozesse. Bad Heilbrunn: Verlag Julius Klinkhardt, S. 338-340.

Wissenschaftsrat (1970). Empfehlungen zur Struktur und zum Ausbau des Bildungswesens im Hochschulbereich nach 1970. Band 2. Anlagen. Bonn: Bundesdruckerei.

Wolter, Jan (2018). Disziplinierungspraktiken in der Grundschule: Formalisierung sozialer Bezugnahme und Egalisierung von Differenz. Anmerkungen zur ,Persönlichkeitsbildung'. In Jürgen Budde \& 